\title{
Determinantes sociais e biológicos da mortalidade infantil no Recôncavo da Bahia
}

\author{
Social and biological determinants of child mortality in the Recôncavo of Bahia
}

\author{
Caroline Nascimento dos Anjos ${ }^{1}$, Carolina Santos Mello², Jerusa da Mota Santana ${ }^{3 *}$
}

\begin{abstract}
${ }^{1}$ Acadêmica do Curso de Graduação em Nutrição pela Universidade Federal da Bahia, UFBA; ${ }^{2}$ Doutora em Ciências pelo Programa de Pediatria e Ciências Aplicadas à Pediatria da Universidade Federal de São Paulo, UNIFESP. Professora Adjunta da Escola de Nutrição da Universidade Federal da Bahia, UFBA; ${ }^{3}$ Doutora em Saúde Pública pelo Instituto de Saúde Coletiva da Universidade Federal da Bahia, UFBA, Professora Adjunta do Centro de Ciências da Saúde, Universidade Federal do Recôncavo da Bahia, UFRB
\end{abstract}

\begin{abstract}
Resumo
Introdução: o Recôncavo é um território de identidade da Bahia propenso a sofrer mais iniquidades sociais, o que pode gerar repercussões negativas nas condições de vida e saúde das crianças e, assim, impactar no Coeficiente de Mortalidade Infantil (CMI). Objetivo: analisar a associação entre os determinantes sociais e biológicos da saúde e a mortalidade infantil nos municípios do Recôncavo da Bahia, no período de 2010 a 2019. Metodologia: estudo ecológico, com dados de Sistemas de Informações em Saúde. Dados de CMI por 1000 nascidos vivos (NV) foram avaliados temporalmente no Recôncavo da Bahia, do ano de 2010 a 2019. Regressão Quantílica foi utilizada para análise do desfecho (CMI) e variáveis de exposição (determinantes). Resultados: o CMI no Recôncavo da Bahia apresentou importantes variações no período avaliado, passando de 9,7/1.000NV em 2010, para 13,1/1.000NV em 2019. Identificou-se associação positiva do CMI com Índice de Gini e uma associação inversa entre o CMI e Índice de Desenvolvimento Humano Municipal (IDHM). Conclusão: no período de 2010 a 2019 houve um aumento de 35\% no CMI, no Recôncavo da Bahia. A mortalidade infantil no Recôncavo da Bahia é determinada socialmente pelo Índice de Gini e IDHM. As condições de saúde das crianças podem sofrer consequências em um contexto de aumento da vulnerabilidade social e políticas de subfinanciamento da saúde. Palavras-chave: Mortalidade de Menores de 1 Ano de Idade. Desigualdades em Saúde. Fatores de Risco. Sistemas de Informação. Saúde da Criança.
\end{abstract}

\begin{abstract}
Introduction: the Recôncavo is an identity territory in Bahia that is prone to suffering more social inequities, which can generate negative repercussions on the living conditions and health of children and, thus, impact on the Infant Mortality Rate (IMR). Objective: to analyze the association between the social and biological determinants of health and infant mortality in the municipalities of Recôncavo of Bahia, in the period 2010-2019. Methodology: ecological study, using data from Health Information Systems. IMR data per 1,000 live births (LB) were temporally evaluated in the Recôncavo of Bahia, from 2010 to 2019. Quantile Regression was used for outcome analysis (IMR) and exposure variables (determinants). Results: the IMR in the Recôncavo of Bahia showed important variations in the evaluated period, going from 9.7/1,000LB in 2010, to 13.1/1,000LB in 2019. A positive association was identified between IMR with Gini Index and an inverse association between the IMR and Municipal Human Development Index (MHDI). Conclusion: in the period of 2010-2019 there was an increase of 35.0\% in the IMR, in the Recôncavo of Bahia. Infant mortality in the Recôncavo of Bahia is socially determined by the Gini Index and MHDI. Children's health conditions can suffer consequences in a context of increased social vulnerability and underfunded health policies.
\end{abstract}

Keywords: Infant Mortality. Health Status Disparities. Risk Factors. Information Systems. Child Health.

\section{INTRODUÇÃO}

A mortalidade infantil consiste nos óbitos ocorridos em menores de um ano de idade, sendo um importante reflexo das condições socioeconômicas, de vida e saúde da população ${ }^{1}$. No Brasil, há pelo menos três décadas, observou-se uma tendência de redução do Coeficiente de Mortalidade Infantil (CMI), que passou de 47,1/1.000 nascidos vivos (NV) em 1990 para 16,7/1.000NV em 2010, e 13,1/1.000NV em 2018². Nesse mesmo período,

Correspondente/Corresponding: *Jerusa da Mota Santana - End.: Av. Carlos Amaral, R. do Cajueiro, 1015, Santo Antônio de Jesus - BA, - CEP: 44574-490 - Tel: (75) 3632-1724 - E-mail: jerusanutri@ufrb.edu.br também houve redução do CMI na região Nordeste e no estado da Bahia².

Avanços que ocorreram no tocante a políticas públicas atuantes nas condições sociais e de saúde ao longo dos últimos anos influenciaram diretamente no decaimento da mortalidade infantil no país, perpassando desde o incentivo ao aleitamento materno, à expansão de programas de transferência condicionada de renda e da Atenção Primária à Saúde ${ }^{3}$. Em 2015, o Brasil juntamente com outros países se tornou signatário dos Objetivos do Desenvolvimento Sustentável (ODS) da Organização das Nações Unidas (ONU), que tem como uma das metas até 2030 acabar com as mortes evitáveis de recém-nascidos e crianças menores de 5 anos $^{4}$. 
Entretanto, apesar dos investimentos em políticas públicas de atenção à saúde materno-infantil, quando comparado com a média regional e nacional, observa-se que o CMI da Bahia permanece com valores superiores ${ }^{2}$, destacando as disparidades inter e intrarregionais existentes.

Entre 2015-2016, após anos de queda, houve um aumento da mortalidade infantil no país e em quatro regiões (Centro-Oeste, Nordeste, Norte e Sudeste) $)^{5}$, o que possivelmente está associado à piora de alguns dos determinantes sociais, como o aumento da pobreza e do subfinanciamento do Sistema Único de Saúde (SUS) a diminuição da cobertura vacinal, o que vem gerando maior incidência de doenças imunopreveníveis ${ }^{6}$, ou até mesmo ter sofrido impacto positivo pela epidemia do vírus Zika, ocorrida em $2015^{7}$.

As iniquidades em saúde podem repercutir negativamente de diferentes formas nas condições de vida das crianças e impactar diretamente no $\mathrm{CMI}$, dentre elas estão as condições de habitação e moradia inadequadas, a baixa renda familiar, a desigualdade de renda, o baixo desenvolvimento humano, a assistência pré-natal e ao parto inadequadas ${ }^{8}$, o que ainda pode contribuir para um aumento na prevalência de insegurança alimentar e nutricional (IA) e assim agravar o número de óbitos infantis ${ }^{9}$. Estes fatores são os Determinantes Sociais da Saúde (DSS), que são características que influenciam na ocorrência de problemas de saúde e seus fatores de risco na população ${ }^{10}$.

O Recôncavo é um dos territórios de identidade da Bahia que apresenta condições incipientes de moradia quando comparados à média estadual, com a maioria dos seus municípios apresentando dependência de transferências do governo federal para programas sociais ${ }^{11}$, além da predominância de $I \mathrm{~A}^{12}$, tornando-o mais suscetível às iniquidades em saúde no atual momento de crise econômica evidenciada pelos cortes de programas de assistência social no país ${ }^{13}$, o que consequentemente tende a gerar ascensão na mortalidade infantil.

Nesse contexto, este estudo tem como objetivo analisar a associação entre os determinantes sociais e biológicos da saúde e a mortalidade infantil nos municípios do Recôncavo da Bahia, no período de 2010-2019.

\section{METODOLOGIA}

Trata-se de um estudo ecológico, cuja unidade de análise são os 19 municípios do Recôncavo da Bahia: Cabaceiras do Paraguaçu, Cachoeira, Castro Alves, Conceição do Almeida, Cruz das Almas, Dom Macedo Costa, Governador Mangabeira, Maragogipe, Muniz Ferreira, Muritiba, Nazaré, Salinas da Margarida, Santo Amaro, Santo Antônio de Jesus, São Felipe, São Félix, Sapeaçu, Saubara e Varzedo ${ }^{11}$. O Recôncavo é um território de identidade localizado na Mesorregião Metropolitana de Salvador, com 514.792 habitantes segundo o Censo $2010^{11}$.

O período escolhido foi de 2010 a 2019, tendo como justificativa a utilização de dados socioeconômicos dis- ponibilizados no Censo Demográfico de 2010, e pela possibilidade de se elaborar uma série temporal do CMI no Recôncavo da Bahia, durante o intervalo de tempo de nove anos.

Considerou-se como variável desfecho o $\mathrm{CMI}$, calculado a partir da divisão do número de óbitos em crianças menores de um determinado ano pelo número de nascidos vivos com residência nos municípios do Recôncavo da Bahia, multiplicando por 1.000 o resultado ${ }^{14}$. Este mesmo cálculo foi empregado individualmente para os componentes do CMI: Coeficiente de Mortalidade Neonatal Precoce (utilizando os óbitos entre 0 a 6 dias de vida), o Coeficiente de Mortalidade Neonatal Tardia (com os óbitos entre 7 a 27 dias), o Coeficiente de Mortalidade Neonatal (óbitos entre 0 a 27 dias) e o Coeficiente de Mortalidade Pós Neonatal (usando os óbitos entre 28 e 364 dias).

As variáveis de exposição foram organizadas de forma hierarquizada em três eixos teóricos por nível de proximidade de ocorrência com o óbito infantil no sentido distal-proximal (Figura 1), baseado nos três níveis incidentes do modelo teórico de Dahlgreen e Whitehead ${ }^{10}$ : determinantes distais, determinantes intermediários e determinantes proximais. As variáveis inseridas em cada bloco foram:

a. Determinantes distais: índice de Gini, razão de renda, renda domiciliar per capita, índice de desenvolvimento humano municipal (IDHM), abastecimento de água (domicílios abastecidos por meio de rede geral de distribuição; poço, nascente ou outras formas de abastecimento), coleta de lixo (domicílios que têm lixo coletado pelo serviço municipal de limpeza; queimado, enterrado, jogado ou outro destino), instalações sanitárias (moradores que têm rede geral de esgoto ou pluvial; fossa séptica, fossa rudimentar, vala, rio/lago/mar, outro escoadouro ou não ter instalação sanitária), cobertura do Programa Bolsa Família (PBF), cobertura de Equipes de Saúde da Família (ESF), cobertura da Atenção Básica (AB), diagnóstico de segurança alimentar e nutricional (SAN) - disponibilidade, acesso, consumo, utilização e geral (segurança alimentar e nutricional; insegurança alimentar e nutricional leve, moderada ou grave), escolaridade materna $(<8 ; \geq 8$ anos), situação conjugal materna (com companheiro; sem companheiro) e raça/cor da criança (branca; amarela; indígena; preta/parda);

b. Determinantes intermediários: número de consultas pré-natal $(<7 ; \geq 7)$, mês de início do pré-natal $(\leq 3 ;>3$ após a concepção), tipo de parto (vaginal; cesáreo) e local de nascimento (hospitalar; não hospitalar);

c. Determinantes proximais: idade materna $(<20$; 20-34; $\geq 35$ anos), tipo de gravidez (única; múltipla), estado nutricional gestacional (baixo peso; eutrofia; excesso de peso), sexo (masculino; feminino), idade gestacional $(<37 ; 37-41 ; \geq 42$ 
semanas), peso ao nascer (<1.500; 1.500-2.499; $\geq 2.500$ gramas), índice de apgar no 10 e 50 minuto de vida $(0-7 ; 8-10)$ e malformação congênita (sim; não).

Os componentes para o cálculo da variável desfecho foram coletados do Painel de Monitoramento de Nascidos Vivos $^{15}$ e do Painel de Monitoramento da Mortalidade Infantil e Fetal ${ }^{16}$. Já as variáveis de exposição do bloco de determinantes distais foram coletadas do Censo Demográfico 2010 no DATASUS ${ }^{17}$, do IBGE Cidades ${ }^{18}$, do MDS - Bolsa Família ${ }^{19}$, do Diagnóstico da Segurança Alimentar e Nutricional do Estado da Bahia ${ }^{12}$, do e-Gestor Atenção Básica ${ }^{20}$, do Painel de Monitoramento de Nascidos Vivos e do Painel de Monitoramento da Mortalidade Infantil e Fetal. As variáveis do bloco de determinantes intermediários foram coletadas do Painel de Mortalidade de Nascidos Vivos. Por fim, as variáveis do bloco de determinantes proximais são provenientes tanto do Painel de Monitoramento de Nascidos Vivos quanto do Sistema de Vigilância Alimentar e Nutricional ${ }^{21}$ e do Painel de Monitoramento da Mortalidade Infantil e Fetal. Ressalta-se que os dados utilizados provenientes dos Painéis de Monitoramento de Nascidos Vivos e da Mortalidade Infantil e Fetal referentes ao ano de 2019, no momento da coleta, constavam como dados preliminares.

Figura 1 - Determinantes da mortalidade infantil com as variáveis do estudo, baseado nos três níveis incidentes do modelo teórico de Dahlgreen e Whitehead ${ }^{10}$.

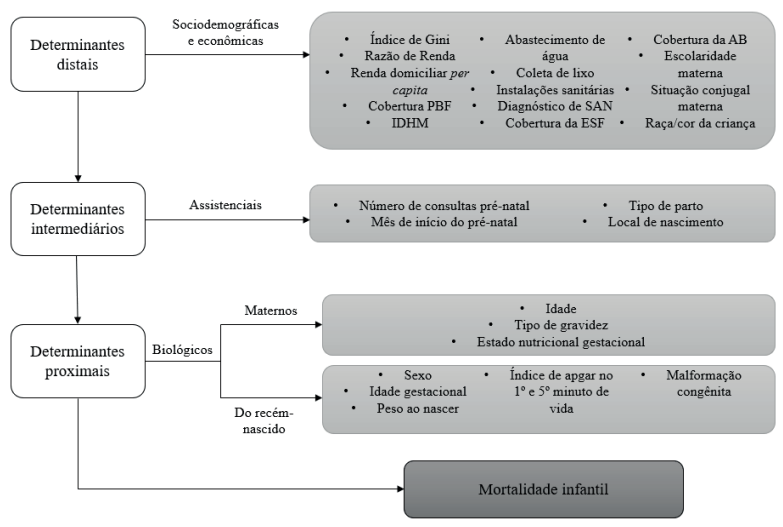

Fonte: Adaptado de Barbosa et al. ${ }^{22}$; Kropiwiec; Franco; Amaral ${ }^{23}$; Lima et al. ${ }^{24}$; Maia; Souza; Mendes ${ }^{25}$; Maia; Souza; Mendes ${ }^{26}$; Martins; Nakamura; Carvalho ${ }^{8}$; Nascimento et al. ${ }^{27}$; Sanders et al. ${ }^{28}$.

Após coleta dos dados, estes foram digitados e analisados no programa Statistical Package For The Social Science for Windows (SPSS), versão 17.0. Realizou-se análise descritiva das variáveis do estudo, aquela de caráter categórico (diagnóstico de SAN) foi analisada por meio de frequências absoluta e relativa, enquanto as variáveis numéricas foram analisadas por meio de média e desvio padrão. Para observar a evolução temporal do CMI empregou-se o gráfico de linhas de séries temporais.

Para quantificar o grau e a intensidade da associação entre as variáveis dependente (CMI) e de exposição (de- terminantes), utilizou-se a Regressão Quantílica. Primeiramente, realizou-se análise bivariada entre as variáveis de exposição e desfecho. Por critério estatístico arbitrário, os autores selecionaram as variáveis para a análise multivariada, mais precisamente as variáveis que apresentaram coeficientes de determinação expressivos (equivalentes a mais de 1 óbito/1.000NV) e as que tinham forte associação teórica com o desfecho ${ }^{26}$. Para as estimativas de medianas do $\mathrm{CMl}$ empregou-se o modelo de regressão quantílica com $\tau=0,5$ (mediana) ${ }^{29}$.

Este estudo utilizou dados secundários de acesso e domínio público, de pesquisa censitária e de pesquisa com bancos de dados cujas informações são agregadas, sem possibilidade de identificação individual, assim a mesma dispensou registro pelo sistema CEP/CONEP.

\section{RESULTADOS}

Segundo o Painel de Monitoramento de Nascidos Vivos, entre 2010-2019, nos municípios do Recôncavo da Bahia nasceram 66.733 crianças, majoritariamente do sexo masculino (51,2\%). No Painel de Monitoramento da Mortalidade Infantil e Fetal foram identificados 627 óbitos infantis, registrados como residentes no mesmo local e período, sendo $55,9 \%$ do sexo masculino.

Na Figura 2, os dados do Coeficiente de Mortalidade Infantil no Recôncavo da Bahia são comparados com os dados do Brasil, do Nordeste e da Bahia, por meio de uma série temporal. O CMI no Recôncavo da Bahia apresentou variações no período 2010-2019, sendo que desde 2016 as taxas se apresentam em ascensão. Em 2010, o CMI era de 9,7/1.000NV, passando para 13,1/1.000NV em 2019, o que representa um acréscimo de $35,0 \%$. De forma contrária, o estado da Bahia (21,0/1.000NV versus 16,7/1.000NV), a região Nordeste (19,1/1.000NV versus $15,0 / 1.000 N V)$ e o Brasil (16,0/1.000NV versus 13,1/1.000NV) apresentaram queda no CMI durante o período 2010-2018, com pequena elevação entre 2015-2016.

Nos municípios do Recôncavo da Bahia, entre 2010 e 2019, o CMI neonatal apresentou taxas muito maiores, quando comparado ao CMI pós-neonatal; e as taxas neonatais precoces, foram superiores às neonatais tardias (Figura 3). O componente neonatal registrou um acréscimo de $33,7 \%$ durante o período estudado, onde o seu coeficiente aumentou de 8,6 para 11,5/1.000NV. 0 componente pós-neonatal apresentou elevação de $60,0 \%$, passando de 1,0 para 1,6/1.000NV, entre 2010 e 2019.

Dentre os determinantes distais relacionados às características econômicas da população do Recôncavo da Bahia registrou-se índice de Gini de 0,55 ( $D P=0,03)$, razão de renda de 28,45 ( $D P=7,57)$, renda domiciliar per capita de $R \$ 329,96(D P=75,76)$ e IDHM de $0,63(D P=0,03)$. A média total de famílias beneficiárias do PBF é de 3.946 ( $D P=2.571)$, com uma cobertura média referente à população total de $37,5 \%(D P=9,3)$. A cobertura média de ESF e da Atenção Básica é de 92,5\% (DP=13,0) e 93,3\% $(\mathrm{DP}=12,1)$, respectivamente. 
Figura 2 - Coeficiente de Mortalidade Infantil (CMI) no Recôncavo da Bahia, no período 2010-2019, e CMI do estado da Bahia, da região Nordeste e do Brasil no período 2010-2018.

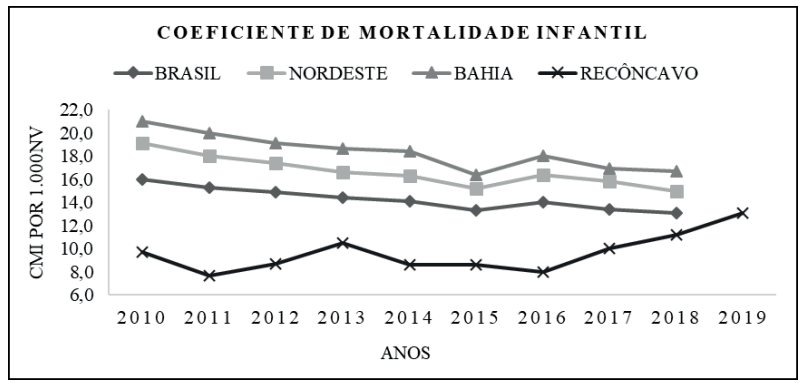

Fonte: Brasil ${ }^{2}$; Painel de Monitoramento de Nascidos Vivos ${ }^{15}$ e Painel da Mortalidade Infantil e Fetal ${ }^{16}$.

Quanto às condições de habitação e moradia deste território de identidade, identificou-se nos determinantes distais (Tabela 1) abastecimento de água e coleta de lixo inadequados em $28,5 \%$ dos domicílios, e instalações sanitárias inadequadas para $67,4 \%$ dos moradores. De acordo com o diagnóstico de SAN revelou-se prevalência, de algum grau de IA, em relação as dimensões: disponibilidade $(94,7 \%)$, acesso $(100 \%)$, consumo $(78,9 \%)$, utilização biológica $(94,7 \%)$ e geral - considerando todas as dimensões (100\%). Dentre as características maternas, observou-se maior registro de mães sem companheiro $(59,5 \%)$ e com pelo menos oito anos de estudo de escolaridade (70,1\%). Dentre as crianças que foram a óbito, $71,6 \%$ eram pretas/pardas.

Identificou-se o predomínio da realização de sete ou mais consultas pré-natal $(55,7 \%)$, com mês de início até aos 3 meses $(68,4 \%)$, maior ocorrência de parto cesáreo $(50,8 \%)$, em ambiente hospitalar $(97,4 \%)$ dentre os determinantes intermediários analisados no presente estudo (Tabela 1).

Figura 3 - Coeficientes de Mortalidade Neonatal Precoce, Neonatal Tardia, Neonatal e Pós-neonatal no Recôncavo da Bahia, 2010-2019.

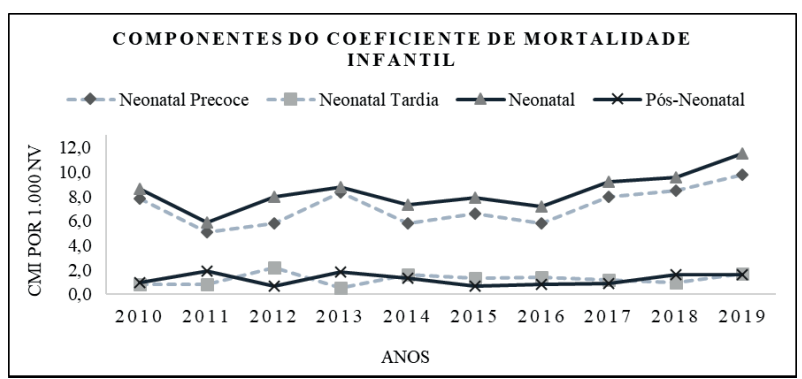

Fonte: Painel de Monitoramento de Nascidos Vivos ${ }^{15}$ e Painel da Mortalidade Infantil e Fetal ${ }^{16}$.

Quanto aos determinantes proximais maternos (Tabela 1), a maioria da população do estudo apresenta idade entre 20 a 34 anos $(69,5 \%)$, estado nutricional de excesso de peso $(44,0 \%)$ diante de uma gravidez única $(97,9 \%)$. Quanto aos fatores proximais da criança, a maioria nasceu entre idade gestacional de 37 a 41 semanas $(79,9 \%)$, com peso ao nascer maior ou igual que $2.500 \mathrm{~g}(92,1 \%)$, sem malformação congênita $(96,4 \%)$, apresentando índice de apgar entre 8 a 10 , tanto no $1^{\circ}$ quanto $5^{\circ}$ minuto de vida (67,4\% e $76,2 \%$, respectivamente).

Tabela 1 - Análise bivariada entre os principais determinantes sociais da saúde e a mortalidade infantil no território de identidade Recôncavo, Bahia, Brasil, 2010-2019.

\begin{tabular}{|c|c|c|c|}
\hline Variáveis & $n(\%)$ & & $\begin{array}{l}\text { Coeficiente } \\
\text { Quantílico* }\end{array}$ \\
\hline \multicolumn{4}{|c|}{ Determinantes distais } \\
\hline Índice de Gini ${ }^{17}$ & & - & $-14,865$ \\
\hline Razão de Renda ${ }^{17}$ & & - & $-0,058$ \\
\hline $\begin{array}{l}\text { Índice de Desenvolvimento } \\
\text { Humano Municipa| }{ }^{18}\end{array}$ & & - & $-3,38$ \\
\hline $\begin{array}{l}\text { Renda Média Domiciliar per } \\
\text { capita }^{17}\end{array}$ & & - & $-0,001$ \\
\hline $\begin{array}{l}\text { Cobertura do PBF referente à } \\
\text { população total }{ }^{19}\end{array}$ & & - & $0,12 \varepsilon$ \\
\hline $\begin{array}{l}\text { Cobertura de Equipes de Saúde da } \\
\text { Família }^{20}\end{array}$ & & - & \\
\hline Cobertura da Atenção Básica²0 & & - & \\
\hline
\end{tabular}

Abastecimento de água ${ }^{17}$

Rede geral de distribuição

$106.648(71,5) \quad-0,000$

Poço, nascente ou outras formas

$42.561(28,5)$

$-0,000$

Coleta de Lixo $^{17}$

Serviço municipal de limpeza

Queimado, jogado, enterrado ou outro destino

$106.638(71,5) \quad-0,000$

$42.571(28,5) \quad-0,000$

Instalações sanitárias ${ }^{17}$

Rede geral de esgoto ou pluvial

Fossa séptica, fossa rudimentar,

vala, rio/lago/mar, outro escoadou-

ro ou não tem instalação sanitária

Diagnóstico de SAN - Disponibilidade ${ }^{12}$

SAN

IA Leve

IA Moderada

IA Grave

Diagnóstico de SAN - Acesso ${ }^{12}$

IA Leve

IA Moderada

$166.928(32,6)$

$-0,000$

$344.816(67,4)$

0

Diagnóstico de SAN - Consumo ${ }^{12}$

SAN

IA Leve

IA Moderada

IA Grave

Diagnóstico de SAN - Utilização ${ }^{12}$

SAN

IA Leve

IA Moderada

$1(5,3)$

$5(26,3)$

$9(47,4)$

$4(21,1)$

$7(36,8)$

$12(63,2)$

$4(21,1)$

$7(36,8)$

$6(31,6)$

$2(10,5)$

$1(5,3)$

$12(63,2)$

$6(31,6)$

Diagnóstico de SAN - Geral ${ }^{12}$

IA Leve

$7(36,8)$

IA Moderada

$12(63,2)$

Escolaridade materna ${ }^{15}$

$<8$ anos de estudo

$17.667(26,5)$

$-0,003$

$\geq 8$ anos de estudo

$46.772(70,1)$

$-0,001$

Situação conjugal materna ${ }^{15}$

Com companheiro

$25.442(38,1)$

$-0,002$

Sem companheiro 


\begin{tabular}{lcc}
\hline \multicolumn{3}{c}{ Tabela 1 (conclusão } \\
\hline Variáveis & $\mathbf{n}(\%)$ & $\begin{array}{l}\text { Coeficiente } \\
\text { Quantílico* }\end{array}$ \\
\hline \multicolumn{3}{c}{ Determinantes distais } \\
\end{tabular}

Raça/Cor da criança ${ }^{16}$
Branca
Amarela
Indígena
Parda/Preta

Determinantes distais

\section{Determinantes intermediários}

Número de consultas pré-natal ${ }^{15}$

$$
\begin{aligned}
& <7 \text { consultas } \\
& \geq 7 \text { consultas }
\end{aligned}
$$

Mês de início do pré-natal ${ }^{15}$

$$
\leq 3 \text { meses }
$$

$>3$ meses

$40.755(68,4)$

$14.043(23,6)$

Tipo de parto ${ }^{15}$

Parto vaginal

Parto cesáreo

$32.678(49,0)$

$33.894(50,8)$

Local de nascimento ${ }^{15}$

$\begin{array}{lrr}\text { Hospitalar } & 64.989(97,4) & -0,000 \\ \text { Não hospitalar } & 1.724(2,6) & 0,035\end{array}$

Idade materna ${ }^{15}$

Determinantes proximais

$<20$ anos

20 a 34 anos

$\geq 35$ anos

$11.951(17,9)$

$46.388(69,5)$

$8.394(12,6)$

$65.306(97,9)$

$1.274(1,9)$

Múltipla

Estado nutricional gestacional ${ }^{21}$

Baixo peso

Eutrofia

Excesso de peso

Sexo $^{15}$

Masculino

Feminino

Idade gestacional ${ }^{15}$

$<37$ semanas

37 a 41 semanas

$>42$ semanas

Peso ao nascer ${ }^{15}$

$$
\begin{aligned}
& <1.500 \mathrm{~g} \\
& 1.500 \text { a } 2.499 \mathrm{~g} \\
& \geq 2.500 \mathrm{~g}
\end{aligned}
$$

$3.535(18,6)$

$7.075(37,3)$

$8.353(44,0)$

$34.195(51,2)$

$32.521(48,7)$

$6.611(9,9)$

$53.342(79,9)$

$3.093(4,6)$

$832(1,2)$

$4.440(6,7)$

$61.449(92,1)$

Índice de apgar no 10 minuto de vida $^{15}$

$$
\begin{aligned}
& 0 \text { a } 7 \\
& 8 \text { a } 10
\end{aligned}
$$

$7.487(11,2)$

$44.992(67,4)$

Índice de apgar no 5으 minuto de vida $^{15}$

$$
0 \text { a } 7
$$$$
8 \text { a } 10
$$$$
1.574(2,4)
$$$$
50.883(76,2)
$$$$
-0,002
$$$$
-0,000
$$

$-0,007$

0,001

$-0,004$

$-0,001$

$-0,044$

$-0,005$

$-0,002$

$-0,001$

$-0,002$

$-0,002$

$-0,010$

0,000

$-0,037$

0,137

$-0,015$

0

Malformação congênita ${ }^{15}$

Sim

$384(0,6)$

$64.323(96,4)$

$-0,001$

Fonte: Sistemas de Informação em Saúde. Dados extraídos em outubro de 2020 , sujeitos às alterações. GGSAN BAHIA ${ }^{12}$; Painel de Monitoramento de Nascidos Vivos ${ }^{15}$; Painel de Monitoramento da Mortalidade Infantil e Fetal ${ }^{16}$; DATASUS ${ }^{17}$; IBGE Cidades ${ }^{18}$; MDS - Bolsa Família ${ }^{19}$;
e-Gestor $\mathrm{AB}^{20}$; Sistema de Vigilância Alimentar e Nutricional ${ }^{21}$. ${ }^{*}$ Regressão quantílica bivariada.

Na análise multivariada, dois determinantes que estão dispostos no nível distal (Índice de Gini e IDHM) apresentaram coeficientes quantílicos considerados importantes, sendo positiva a associação entre CMI e Índice de Gini e

\begin{tabular}{|c|c|}
\hline Variáveis & $\begin{array}{l}\text { Coeficiente } \\
\text { Quantílico* }\end{array}$ \\
\hline \multicolumn{2}{|l|}{ Determinantes distais } \\
\hline Índice de Gini ${ }^{17}$ & 13,208 \\
\hline Índice de Desenvolvimento Humano Municipal ${ }^{18}$ & $-16,079$ \\
\hline \multicolumn{2}{|l|}{ Escolaridade materna ${ }^{15}$} \\
\hline$<8$ anos de estudo & 0,000 \\
\hline$\geq 8$ anos de estudo & - \\
\hline \multicolumn{2}{|l|}{ Situação conjugal materna ${ }^{15}$} \\
\hline Com companheiro & - \\
\hline Sem companheiro & 0,012 \\
\hline \multicolumn{2}{|l|}{ Determinantes intermediários } \\
\hline \multicolumn{2}{|l|}{ Número de consultas pré-natal ${ }^{15}$} \\
\hline$<7$ consultas & 0,004 \\
\hline$\geq 7$ consultas & - \\
\hline \multicolumn{2}{|l|}{ Determinantes proximais } \\
\hline \multicolumn{2}{|l|}{ Idade materna ${ }^{15}$} \\
\hline$<20$ anos & $-0,053$ \\
\hline 20 a 34 anos & - \\
\hline$\geq 35$ anos & - \\
\hline \multicolumn{2}{|l|}{ Tipo de gravidez $z^{15}$} \\
\hline Única & - \\
\hline Múltipla & $-0,220$ \\
\hline \multicolumn{2}{|l|}{ Sexo $^{15}$} \\
\hline Masculino & $-0,013$ \\
\hline Feminino & - \\
\hline \multicolumn{2}{|l|}{ Idade gestacional $\left.\right|^{15}$} \\
\hline$<37$ semanas & $-0,020$ \\
\hline 37 a 41 semanas & - \\
\hline$>42$ semanas & - \\
\hline \multicolumn{2}{|l|}{ Peso ao nascer ${ }^{15}$} \\
\hline$<1.500 \mathrm{~g}$ & 0,776 \\
\hline 1.500 a $2.499 \mathrm{~g}$ & $-0,007$ \\
\hline$\geq 2.500 \mathrm{~g}$ & - \\
\hline \multicolumn{2}{|l|}{ Índice de apgar no 19 minuto de vida ${ }^{15}$} \\
\hline 0 a 7 & 0,005 \\
\hline 8 a 10 & - \\
\hline \multicolumn{2}{|l|}{ Malformação congênita ${ }^{15}$} \\
\hline Sim & 0,017 \\
\hline Não & - \\
\hline
\end{tabular}
inversa a associação entre CMI e IDHM. Assim, observou-se que a cada aumento em um ponto na escala do Índice de Gini estimou-se um aumento mediano de 13 óbitos por 1.000NV, e a cada aumento na escala do IDHM estimou-se uma redução mediana de 16 óbitos por 1.000NV (Tabela 2).

Tabela 2 - Associação entre os determinantes sociais da saúde e a mortalidade infantil no território de identidade Recôncavo, Bahia, Brasil, 2010-2019

Fonte: Sistemas de Informação em Saúde. Dados extraídos em outubro de 2020, sujeitos às alterações. Painel de Monitoramento de Nascidos Vivos $^{15}$; DATASUS ${ }^{17}$; IBGE Cidades ${ }^{18} *$ Regressão quantílica multivariada. 


\section{DISCUSSÃO}

Neste estudo foi possível observar menor Coeficiente de Mortalidade Infantil no Recôncavo da Bahia quando comparado a nível estadual, regional e nacional, no período de 2010 a 2018, apesar deste apresentar aumento desde o ano de 2016. Verificou-se que Índice de Gini e IDHM (determinantes distais) implicaram em associação positiva e inversa com o $\mathrm{CMI}$, respectivamente.

Observa-se no período 2015-2016, após anos de queda das taxas, um aumento da mortalidade infantil no Brasil, no Nordeste e na Bahia, seguido por uma redução nos anos seguintes. De forma contrária, o CMI do Recôncavo apresentou um comportamento variável e não se elevou entre 2015-2016; contudo, diferentemente dos demais níveis de abrangência, este se manteve crescente a partir de 2016, o que pode indicar um agravamento de problemas na assistência à saúde materno-infantil no contexto de implantação da Emenda Constitucional 95 que congelou os gastos da União com serviços primários - incluindo a saúde - até $2036^{13}$.

Os cortes ocorridos no SUS têm como consequência o comprometimento da sustentabilidade de todo o sistema de saúde, afetando a ocorrência dos óbitos infantis. A falta de integração entre as ações desenvolvidas na atenção primária e os serviços de atenção ao parto também contribuem para falhas na linha de cuidado da gestante ${ }^{5}$.

Medidas para garantia da assistência pré-natal e parto seguro são importantes, mas não suficientes, uma adequada assistência ao nascimento e garantia de condições do aleitamento materno são exemplos de medidas a serem aprimoradas no país. As políticas elaboradas para a redução da mortalidade infantil devem focar não apenas na criança em si, como também na mulher, que desempenha papel crucial no cuidado da criança ${ }^{1}$. Um cuidado centrado na pessoa tendo como princípios: ser personalizado, coordenado, capacitante e oferecido com dignidade, compaixão e respeito ${ }^{30}$, quando fornecido à mãe tem o potencial de gerar um menor risco de vida das crianças.

O CMI neonatal apresentou valores maiores que o CMI pós-neonatal, destacando-se o componente neonatal precoce. Este achado é reiterado por diversos autores em outros locais do país $22-24,28$, evidenciando a necessidade de melhoria na assistência no ciclo gravídico-puerperal.

$\mathrm{O} \mathrm{CMI}$ é um indicador que se relaciona fortemente com as iniquidades em saúde ${ }^{1}$, assim, o seu aumento pode estar associado a inúmeros fatores influenciadores das condições de vida que vem sendo agravados no país, a exemplo do aumento da pobreza e do subfinanciamento do SUS ${ }^{5}$. Tal fato, consequentemente, pode interferir nas condições de habitação e moradia da população, além do acesso aos serviços de saúde, gerando impacto sobre a equidade em saúde e o bem-estar.

A epidemia de vírus Zika ocorrida em $2015^{7}$ também pode ter impactado o $\mathrm{CMI}$ por meio do aumento do número de óbitos infantis pós-neonatal e da proporção de mulheres que evitaram a gravidez no período ${ }^{5}$.

Todo esse cenário se torna ainda mais preocupante com a implementação de medidas de austeridade fiscal no Brasil que vem sendo feita diante do cenário de crise econômica enfrentado desde 2014, podendo gerar um aumento substancial na morbidade e mortalidade infantil até $\mathrm{o}$ ano de 2030, mediante cortes de programas como o PBF e a ESF ${ }^{13}$, ameaçando o cumprimento dos ODS para a saúde infantil.

Além disso, o atual contexto de pandemia causada pela Covid-19 pode reverter a redução dessa mortalidade diante das interrupções nos serviços de saúde enfrentados por todo o mundo ${ }^{31}$, dentre elas serviços de imunização, exames pré-natais e cuidados pós-natais, essenciais para evitar mortes nessa faixa etária ${ }^{32}$. Não obstante, a crise socioeconômica global causada pela pandemia pode resultar num aumento da situação de pobreza em famílias com crianças, sem acesso a serviços essenciais, em ausência de quaisquer políticas atenuantes ${ }^{32}$.

No Recôncavo da Bahia os indicadores de concentração de renda apresentam tendência a desnível de renda entre grupos populacionais, demonstrando desigualdade. Encontrou-se uma associação entre óbitos infantis com o Índice de Gini e IDHM. Ou seja, quanto maior a desigualdade de renda e menor o desenvolvimento humano nos municípios do Recôncavo da Bahia, maior é o CMI. A renda média domiciliar per capita é baixa, e o índice de desenvolvimento humano dos municípios se apresentam piores que o índice estadual, com exceção do IDHM de Cruz das Almas e Santo Antônio de Jesus ${ }^{11}$.

A desigualdade de renda, baixa renda média domiciliar per capita e o baixo desenvolvimento humano caracterizam um estado de vulnerabilidade social para as crianças desse território de identidade, o que dificulta e fragiliza acesso a direitos como moradia adequada, saneamento básico, serviços de saúde ${ }^{8}$ e especialmente, a renda familiar ao afetar as suas condições de saúde por meio do acesso aos alimentos e a qualidade destes ${ }^{33}$. Todos esses fatores podem facilitar ou dificultar uma situação de Segurança Alimentar e Nutricional, consagrada com o seguinte conceito ${ }^{34}$ :

Consiste na realização do direito de todos ao acesso regular e permanente a alimentos de qualidade, em quantidade suficiente, sem comprometer o acesso a outras necessidades essenciais, tendo como base práticas alimentares promotoras da saúde, que respeitem a diversidade cultural e que sejam ambiental, cultural, econômica e socialmente sustentáveis (BRASIL, 2006, Art. $\left.3^{\circ}\right)$.

O Recôncavo da Bahia está inserido num contexto em que o seu estado e região apresentam prevalências de IA acima da média nacional ${ }^{9}$. Dados do cenário nacional provenientes da Pesquisa de Orçamentos Familiares (POF) 2017-2018 ${ }^{35}$ registram proporção de domicílios em IA Leve de $24,0 \%$, em IA Moderada 8,1\% dos domicílios e $4,6 \%$ dos domicílios em IA Grave. Ou seja, em algum momento durante um período de 3 meses houve mudança 
nos padrões de alimentação e a fome esteve presente nesses domicílios.

A extinção do Conselho Nacional de Segurança Alimentar e Nutricional (CONSEA) por meio da Medida Provisória ${ }^{\circ} 870$ no dia 01/01/2019 é um fator relevante nesse contexto de IA, pois fragilizou de maneira profunda o funcionamento do Sistema Nacional de Segurança Alimentar e Nutricional (SISAN), comprometendo processos de garantia do Direito Humano à Alimentação Adequada (DHAA) em todas as esferas de governo ${ }^{36}$. Fato este ainda mais preocupante no cenário de crise econômica com aplicação de medidas de austeridade fiscal sendo aplicadas no país ${ }^{36}$, e diminuição da proporção dos domicílios em situação de SAN existente, independente da região, concomitante com o aumento expressivo de todos os graus associados à situação de $\mathrm{I} A$, que vinham num cenário de redução em todo o país ${ }^{35}$.

Nessa perspectiva, este estudo encontrou uma elevada prevalência de IA geral e nas seguintes dimensões "Disponibilidade", "Acesso", "Consumo", "Utilização". Se torna alarmante resultados como este, uma vez que a POF 2017-2018 ${ }^{35}$ revela que, a alimentação ocupa o $3^{\circ}$ lugar nas despesas de consumo nos domicílios, logo atrás de gastos com habitação e transporte, se tornando menor ainda essa despesa à medida em que a situação de SAN progride para IA e esta vai se agravando, chamando atenção para possíveis dificuldades em garantir o acesso a alimentação adequada. Com relação as despesas com alimentação, percebe-se que a maioria dos gastos entre os grupos de alimentos diminui conforme aumenta os níveis de IA sendo encontradas diferenças expressivas no grupo das Frutas; Legumes e verduras; Carnes, vísceras e pescados; Leites e derivados.

As condições de saneamento básico apresentam associações fortemente evidenciadas na literatura científica com a mortalidade infanti| $\left.\right|^{8,37}$. Domicílios em situação de IA apresentam condições de saneamento básico inferiores àqueles em situação de SAN, se agravando quanto maior for o nível de $\mathrm{IA}^{35}$. A baixa escolaridade materna comumente se associa aos óbitos infantis ${ }^{25,26}$, sendo a educação outro direito indispensável aos indivíduos para a plena realização da $\mathrm{SAN}^{12}$.

Acesso a serviços relacionados à saúde na atenção básica pela ESF é primordial para a melhoria dos indicadores de saúde e nutrição da população e, consequentemente, a promoção da $\mathrm{SAN}^{12}$. No tocante aos óbitos infantis, a ESF exerce influência positiva no desenvolvimento das crianças, auxiliando na redução da mortalidade infantil ao longo dos anos a partir do momento em que o município adere a estratégia, gerando uma influência positiva na promoção do aleitamento materno, cuidados pré-natais e neonatais, imunização e o controle de doenças infectocontagiosas ${ }^{38}$.

Ao analisar a interação entre a ESF e outros programas sociais, como o PBF, seu efeito é ainda maior na melhoria da saúde das crianças, podendo se complementarem mediante os diferentes aspectos de riscos que ambos lidam ${ }^{38}$. Sua potencialidade está associada aos condicionantes necessários para que as famílias possam receber os benefícios, como exames pré-natais, orientações sobre saúde e nutrição, vacinação e frequência escolar. Nesse sentido, estudos mostram que o PBF possui efeito sobre a mortalidade infantil, independente da presença da $\mathrm{ESF}^{22,37}$, entretanto, quando ambos estão presentes, esses efeitos são potencializados ${ }^{38}$. Ainda, a ampliação da renda por meio do PBF gera uma melhoria dos níveis de SAN/ IA leve e dos indicadores socioeconômicos ${ }^{33}$, destacando assim a importância de políticas e programas atuantes nos determinantes distais.

Dessa forma, diante de todas as conquistas obtidas por essas políticas e programas sociais, é necessário evitar cortes orçamentários que gerem ainda mais retrocessos à saúde da população, especialmente a infantil. Uma estagnação das transferências aos municípios pode reverter parcialmente algumas das conquistas obtidas nas últimas décadas, aumentando a desigualdade entre municípios em relação à mortalidade infantil ${ }^{38}$.

A população negra está entre os segmentos mais afetados pela $I \mathrm{~A}^{35}$, demonstrando disparidades étnico-raciais nas condições que dão forma à situação de SAN/ IA na população, juntamente, há maior vulnerabilidade à restrição alimentar nos domicílios onde residem crianças/ adolescentes ${ }^{35}$. Esses grupos estão expostos a um maior risco, podendo existir um agravamento na relação raça/ cor negra versus IA versus mortalidade infantil.

No Brasil, crianças brancas e amarelas apresentam menores valores de $\mathrm{CMl}$ enquanto os indígenas apresentam CMI mais elevado do que os demais segmentos da população brasileira, se aproximando do segmento preto reconhecidamente desfavorecido quando comparados aos demais grupos de raça/cor ${ }^{27}$, confirmando a existência de desigualdades étnico-raciais. Especificamente quanto a população preta/parda, esta apresentou maior risco de morte no primeiro ano de vida em Recife e Porto Alegre ${ }^{25}$, em contraponto ao estudo com crianças de Cuiabá em que esta cor da pele não demonstrou associação comparando-as com a cor branca ${ }^{24}$, demonstrando que apesar das crianças não brancas frequentemente irem mais a óbito, as diferenças locais dessa variável bem como a sua categorização podem influenciar na sua significância.

Apesar de não se associarem com o CMI neste estudo, o baixo peso ao nascer e a prematuridade são fatores que apresentam uma interação sugestiva na mortalidade infantil, principalmente para o óbito neonata ${ }^{27}$, mediante o fato que a evolução temporal da gestação é acompanhada pelo aumento progressivo de peso do recém-nascido ${ }^{23}$.

O crescimento intrauterino restrito (CIUR) é apontado como uma das possíveis causas do baixo peso ao nascer ${ }^{40}$. Para o muito baixo peso ao nascer, especificamente, existe uma associação dessa classe de nascidos com a baixa renda familiar ${ }^{41}$. Isso reflete, de certa forma, uma interação complexa entre fatores biológicos, as condições socioeconômicas e a qualidade da assistência prestada à mãe durante a gestação ${ }^{28,40}$, e podem conferir maiores 
chances de morbidades não só no primeiro ano de vida da criança, aumentando o risco de óbito ${ }^{22}$, como também contribuir no surgimento de doenças em ciclos posteriores da vida, fenômeno denominado programação ou hipótese da origem fetal de doenças ${ }^{42}$.

O índice de apgar é uma maneira de avaliar a vitalidade dos recém-nascidos ${ }^{26}$, sendo um baixo valor indicativo de ocorrência de asfixia na criança ${ }^{25}$. Quanto menor o índice em ambas as situações, menores as chances de sobrevivência da criança, o que reforça a importância de uma assistência adequada especialmente no parto, como também na gestação e no pós-parto ${ }^{23,25,40}$.

Em síntese, este estudo permitiu identificar que a mortalidade infantil no Recôncavo da Bahia é influenciada pelos determinantes sociais Índice de Gini e IDHM. Todos esses resultados contribuem para uma melhor compreensão dos DSS nas condições de saúde da população infantil residente no local, de forma que intervenções eficazes abrangendo os diferentes níveis de determinantes possam ser feitas para a geração de indicadores de saúde mais satisfatórios. A oferta adequada de serviços essenciais como geração de renda e emprego, alimentação e saúde, assim como o fortalecimento de políticas públicas de desenvolvimento regional podem contribuir para um melhor desfecho gestacional e impactar positivamente a saúde das crianças e, consequentemente, o CMI.

Ressalta-se que os dados de CMI a nível estadual, regional e nacional foram obtidos pelo site do Departamento de Análise de Saúde e Vigilância de Doenças Não Transmissíveis, do Ministério da Saúde², que utiliza a metodologia do Busca Ativa, estando estes disponíveis até o ano de 2018, diferentemente do CMI do Recôncavo, calculado com base nos dados coletados de nascidos vivos e óbitos residentes até o ano de 2019. Ainda, a utilização de fontes de dados secundários torna-se um fator limitante diante da imprecisão e incompletude que estes podem apresentar.

Outras limitações deste estudo provêm da falácia ecológica, pois esses achados de efeito ecológico não necessariamente refletem o efeito biológico a nível individual. Destaca-se como potencialidade para estudos futuros a realização da busca ativa de casos subnotificados no local do estudo, juntamente com a obtenção de dados primários, devido aos questionamentos existentes acerca da qualidade dos dados provenientes dos Sistemas de Informação em Saúde.

\section{CONCLUSÃO}

A mortalidade infantil no Recôncavo da Bahia é determinada socialmente pelo Índice de Gini e IDHM. É preocupante a influência que estes determinantes podem exercer sobre as condições de saúde das crianças em um local de vulnerabilidade social, em que a mortalidade infantil se encontra em ascensão, especialmente em um contexto de crise econômica, com cortes de programas sociais e o atual cenário de crise sanitária, gerando o agravamento dos diferentes DSS.

O acompanhamento temporal da mortalidade infantil assim como o conhecimento dos fatores associados aos óbitos infantis pode contribuir no processo de implementação e melhoria de políticas públicas já existentes, tornando-as mais eficazes no intuito de atender problemas de ordem social e assistencial que atingem a população, principalmente grupos mais vulneráveis como o materno-infantil e, consequentemente, possam reduzir o risco de morte das crianças.

\section{REFERÊNCIAS}

1. BRASIL. Ministério da Saúde. Secretaria de Vigilância em Saúde. Departamento de Vigilância de Doenças e Agravos Não Transmissíveis e Promoção da Saúde. Saúde Brasil 2015/2016: uma análise da situação de saúde e da epidemia pelo vírus Zika e por outras doenças transmitidas pelo Aedes aegypti. Brasília: Ministério da Saúde, 2017. Disponível em: http://svs.aids.gov.br/dantps/centrais-de-conteudos/ publicacoes/saude-brasil/saude-brasil-2015-2016-uma-analise-da-situacao-de-saude-e-da-epidemia-pelo-virus-Zika-e-por-outras-doencas-transmitidas-pelo-Aedes-aegypti.pdf. Acesso em: 16 out. 2020.

2. BRASIL. Ministério da Saúde. Secretaria de Vigilância em Saúde. Departamento de Análise de Saúde e Vigilância de Doenças Não Transmissíveis. Indicadores de mortalidade que utilizam a metodologia do Busca Ativa. Brasília: Ministério da Saúde, 2020. Disponível em: http:// svs.aids.gov.br/dantps/acesso-a-informacao/acoes-e-programas/busca-ativa/indicadores-de-saude/mortalidade/. Acesso em: 16 out. 2020.

3. INSTITUTO DE PESQUISA ECONÔMICA APLICADA. Objetivos de Desenvolvimento do Milênio: Relatório Nacional de Acompanhamento. Brasília: IPEA, 2014. Disponível em: http://www.ipea.gov.br/ portal/images/stories/PDFs/140523_relatorioodm.pdf. Acesso em: 16 out. 2020.

4. INSTITUTO DE PESQUISA ECONÔMICA APLICADA. Objetivos de Desenvolvimento Sustentável. Objetivo 3 - Boa Saúde e Bem-Estar. 2019. Disponível em: https://www.ipea.gov.br/ods/. Acesso em: 31 out. 2020.

5. ASSOCIAÇÃO BRASILEIRA DE SAÚDE COLETIVA. Especial Abrasco sobre o aumento da mortalidade infantil e materna no Brasil. 2018. Disponível em: https://www.abrasco.org.br/site/noticias/institucional/especial-abrasco-sobre-o-aumento-da-mortalidade-infantil-e-materna-no-brasil/36777/. Acesso em: 22 out. 2020.

6. SATO, A. P. S. Qual a importância da hesitação vacinal na queda das coberturas vacinais no Brasil?. Rev. Saúde Pública, São Paulo, v. 52, n. 96, 2018. Disponível em: https://www.scielo.br/pdf/rsp/v52/pt_00348910-rsp-52-87872018052001199.pdf. Acesso em: 16 out. 2020.

7. HENRIQUES, C. M. P.; DUARTE, E.; GARCIA, L. P. Desafios para o enfrentamento da epidemia de microcefalia. Epidemiol. Serv. Saúde, Brasília, v. 25, n. 1, p. 7-10, mar. 2016. Disponível em: https://www.scielo. br/scielo.php?script=sci_arttext\&pid=S2237-96222016000100007. Acesso em: 16 out. 2020.

8. MARTINS, I. P. M.; NAKAMURA, C. Y.; CARVALHO, D. R. Variáveis associadas à mortalidade materna e infantil: uma revisão integrativa. Rev. de Atenção à Saúde, São Caetano do Sul, SP, v.1, n. 64, p. 145-165, abr./jun. 2020. Disponível em: https://webcache.googleusercontent.com/search?q=cache:PoUZn8geEAsJ:https://seer.uscs. edu.br/index.php/revista_ciencias_saude/article/download/6576/ pdf $/ 21727+\& c d=3 \& h l=p t-B R \& c t=c l n k \& g l=b r$. Acesso em: 16 out. 2020.

9. BEZERRA, M. S. et al. Insegurança alimentar e nutricional no Brasil e sua correlação com indicadores de vulnerabilida- 
de. Ciênc. Saúde Colet., Rio de Janeiro, v. 25, n. 10, p. $3833-$ 3846, out. 2020. Disponível em: https://www.scielo.br/scielo. php?pid=S1413-81232020001003833\&script=sci_arttext. Acesso em: 16 out. 2020.

10. BUSS, P. M.; PELLEGRINI FILHO, A. A saúde e seus determinantes sociais. Physis (Rio J.), Rio de Janeiro, v. 17, n. 1, p.77-93, abr. 2007. Disponível em: http://www.scielo.br/pdf/physis/v17n1/v17n1a06.pdf. Acesso em: 16 out. 2020.

11. SUPERINTENDÊNCIA DE ESTUDOS ECONÔMICOS E SOCIAIS DA BAHIA. Perfil dos Territórios de Identidade da Bahia. Salvador: SEI, 2016. v. 2.

12. GRUPO GOVERNAMENTAL DE SEGURANÇA ALIMENTAR E NUTRICIONAL. Diagnóstico da Segurança Alimentar e Nutricional do Estado da Bahia. Bahia: GGSAN, 2015. Disponível em: http://www.casacivil.ba.gov. br/arquivos/File/GGSanDiagnosticodaSegurancaAlimentareNutricionaldoEstadodaBahia2015.pdf. Acesso em: 16 out. 2020.

13. RASELLA, D. et al. Child morbidity and mortality associated with alternative policy responses to the economic crisis in Brazil: A nationwide microsimulation study. Plos Med., San Francisco, v. 15, n. 5, p. 1-20, 2018. Disponível em: https://journals.plos.org/plosmedicine/ article?id=10.1371/journal.pmed.1002570. Acesso em: 16 out. 2020.

14. DATASUS. Indicadores de mortalidade. Disponível em: http:// tabnet.datasus.gov.br/cgi/idb2000/fqc01.htm. Acesso em: 18 fev. 2021.

15. BRASIL. Ministério da Saúde. Secretaria de Vigilância em Saúde. Departamento de Análise de Saúde e Vigilância de Doenças Não Transmissíveis. Painel de Monitoramento de Nascidos Vivos. Disponível em: http://svs.aids.gov.br/dantps/centrais-de-conteudos/paineis-de-monitoramento/natalidade/nascidos-vivos/. Acesso em: 30 set. 2020.

16. BRASIL. Ministério da Saúde. Secretaria de Vigilância em Saúde. Departamento de Análise de Saúde e Vigilância de Doenças Não Transmissíveis. Painel de Monitoramento da Mortalidade Infantil e Fetal. Disponível em: http://svs.aids.gov.br/dantps/centrais-de-conteudos/ paineis-de-monitoramento/mortalidade/infantil-e-fetal/. Acesso em: 30 set. 2020.

17. BRASIL. Ministério da Saúde. DATASUS. Informações de Saúde (TABNET) - Demográficas e Socioeconômicas. Disponível em: http:// www2. datasus.gov.br/DATASUS/index. php?area $=0206 \& i d=6947$. Acesso em: 30 set. 2020.

18. IBGE CIDADES. Índice de Desenvolvimento Humano Municipal. Disponível em: https://cidades.ibge.gov.br/. Acesso em: 30 set. 2020.

19. BRASIL. Ministério do Desenvolvimento Social e Combate à Fome. Programa Bolsa Família. Disponível em: https://aplicacoes.mds.gov.br/ sagirmps/bolsafamilia/. Acesso em: 30 set. 2020.

20. BRASIL. Ministério da Saúde. Secretaria de Atenção Primária à Saúde. e-Gestor Atenção Básica - Informação e Gestão da Atenção Básica. Disponível em: https://egestorab.saude.gov.br/paginas/acessoPublico/ relatorios/relatoriosPublicos.xhtml. Acesso em: 30 set. 2020.

21. BRASIL. Ministério da Saúde. Secretaria de Atenção Primária à Saúde. Sistema de Vigilância Alimentar e Nutricional. Disponível em: https://sisaps.saude.gov.br/sisvan/relatoriopublico/index. Acesso em: 30 set. 2020.

22. BARBOSA, T. A. G. da S. et al. Determinantes da mortalidade infantil em municípios do Vale do Jequitinhonha, Minas Gerais, Brasil. REME Rev. Min. Enferm., Belo Horizonte, v. 18, n. 4, p. 907-914, out./dez. 2014. Disponível em: https://www.reme.org.br/artigo/detalhes/972. Acesso em: 21 out. 2020.

23. KROPIWIEC, M. V.; FRANCO, S. C.; AMARAL, A. R. do. Fatores associados à mortalidade infantil em município com Índice de Desenvolvimento
Humano elevado. Rev. Paul. Pediatr., São Paulo, v. 35, n. 4, p. 391-398, set. 2017. Disponível em: https://www.scielo.br/pdf/rpp/v35n4/01030582-rpp-2017-35-4-00006.pdf. Acesso em: 21 out. 2020.

24. LIMA, J. C. et al. Estudo de base populacional sobre mortalidade infantil. Ciênc. Saúde Coletiva, Rio de Janeiro, v. 22, n. 3, p. 931-939, mar. 2017. Disponível em: http://www.scielo.br/scielo.php?script=sci_ arttext\&pid=S1413-81232017002300931\&Ing=en\&nrm=iso. Acesso em: 21 out. 2020.

25. MAIA, L. T. de S.; SOUZA, W. V. de; MENDES, A. da C. G. Diferenciais nos fatores de risco para a mortalidade infantil em cinco cidades brasileiras: um estudo de caso-controle com base no SIM e no SINASC. Cad. Saúde Pública, Rio de Janeiro, v. 28, n. 11, p. 2163-2176, nov. 2012. Disponível em: http://www.scielo.br/scielo.php?script=sci_arttext\&pid=S0102-311X2012001100016\&lng=en\&nrm=iso. Acesso em: 21 out. 2020.

26. MAIA, L. T. de S.; SOUZA, W. V. de; MENDES, A. da C. G. Determinantes individuais e contextuais associados à mortalidade infantil nas capitais brasileiras: uma abordagem multinível. Cad. Saúde Pública, Rio de Janeiro, v. 36, n. 2, e00057519, 2020. Disponível em: http://www. scielo.br/scielo.php?script=sci_arttext\&pid=S0102-311X20200002050 $12 \&$ Ing=en\&nrm=iso. Acesso em: 21 out. 2020.

27. NASCIMENTO, R. M. do et al. Determinantes da mortalidade neonatal: estudo caso-controle em Fortaleza, Ceará, Brasil. Cad. Saúde Pública, Rio de Janeiro, v. 28, n. 3, p. 559-572, mar. 2012. Disponível em: http://www.scielo.br/scielo.php?script=sci_arttext\&pid=S0102-311X20 $12000300016 \&$ lng=en\&nrm=iso. Acesso em: 21 out. 2020.

28. SANDERS, L. S. de C. et al. Mortalidade infantil: análise de fatores associados em uma capital do Nordeste brasileiro. Cad. Saúde Colet., Rio de Janeiro, v. 25, n. 1, p. 83-89, mar. 2017. Disponível em: http:// www.scielo.br/scielo.php?script=sci_arttext\&pid=S1414-462X2017000 100083\&lng=en\&nrm=iso. Acesso em: 21 out. 2020.

29. KOENKER, R.; BASSETT JUNIOR, G. Regression quantiles. Econometrica, v. 46, n. 1, p. 33-50, jan. 1978. Disponível em: https://www.jstor. org/stable/1913643. Acesso em: 18 jun. 2021.

30. PROQUALIS. Simplificando o cuidado centrado na pessoa: o que todos devem saber sobre o cuidado centrado na pessoa. Rio de Janeiro: PROQUALIS, 2016. Disponível em: https://proqualis.net/sites/proqualis. net/files/Simplificando-o-cuidado.pdf. Acesso em: 30 nov. 2020.

31. WORLD HEALTH ORGANIZATION. Pulse survey on continuity of essential health services during the COVID-19 pandemic: Interim report. Geneva, Aug. 2020. Disponível em: https://www.who.int/ publications/i/item/WHO-2019-nCoV-EHS_continuity-survey-2020.1. Acesso em: 30 nov. 2020.

32. UNICEF. COVID-19 and children. Oct. 2020. Disponível em: https:// data.unicef.org/covid-19-and-children/?utm_source=UNICEF\%20 Data\&utm_medium=homepage\&utm_campaign=COVID19-hub-unicef-data-hero-image. Acesso em: 30 nov. 2020.

33. CABRAL, C. S. et al. Segurança alimentar, renda e Programa Bolsa Família: Estudo de coorte em municípios do interior da Paraíba, Brasil, 2005-2011. Cad. Saúde Pública, Rio de Janeiro, v. 30, n. 2, p. 393-402, fev. 2014. Disponível em: http://www.scielo.br/scielo. php? script=sci_arttext\&pid=S0102-311X2014000200393\&lng=en\&nr $\mathrm{m}=$ iso. Acesso em: 22 out. 2020.

34. BRASIL. Lei n. 11.346, de 15 de Setembro de 2006. Cria o Sistema Nacional de Segurança Alimentar e Nutricional - SISAN com vistas em assegurar o direito humano à alimentação adequada e dá outras providências. Brasília: Presidência da República, 2006. Disponível em: http://www.planalto.gov.br/ccivil_03/_ato2004-2006/2006/lei//11346. htm\#: :text=Cria\%20०\%20Sistema\%20Nacional\%20de,adequada\%20 e\%20d\%C3\%A1\%20outras\%20provid\%C3\%AAncias. Acesso em: 21 out. 2020. 
35. IBGE. Coordenação de Trabalho e Rendimento. Pesquisa de orçamentos familiares 2017-2018: análise da segurança alimentar no Brasil. Rio de Janeiro: IBGE, 2020. Disponível em: https://biblioteca.ibge.gov. $\mathrm{br} /$ index. php/biblioteca-catalogo?view=detalhes\&id=2101749. Acesso em: 14 nov. 2020.

36. CASTRO, I. R. R. de. A extinção do Conselho Nacional de Segurança Alimentar e Nutricional e a agenda de alimentação e nutrição. Cad. Saúde Pública, Rio de Janeiro, v. 35, n. 2, e00009919, 2019. Disponível em: http://www.scielo.br/scielo.php?script=sci_arttext\&pid=S0102-311X20 19000200101\&lng=en\&nrm=iso. Acesso em: 14 nov. 2020.

37. SILVA, E. S. de A. da; PAES, N. A. Programa Bolsa Família e a redução da mortalidade infantil nos municípios do Semiárido brasileiro. Ciênc. Saúde Coletiva, Rio de Janeiro, v. 24, n. 2, p. 623-630, fev. 2019. Disponível em: http://www.scielo.br/scielo.php?script=sci_arttext\&pid=S1413$-81232019000200623 \& \operatorname{lng}=e n \& n r m=i s o$. Acesso em: 21 out. 2020.

38. COMITÊ CIENTÍFICO NÚCLEO CIÊNCIA PELA INFÂNCIA. Impactos da estratégia saúde da família e desafios para o desenvolvimento infantil: estudo 5. São Paulo: NCPI, 2019. Disponível em: https://ncpi.org.br/ wp-content/uploads/2019/12/AF_NCPI-WP_n5_2019_online_v2.pdf. Acesso em: 21 out. 2020.
39. CALDAS, A. D. R. et al. Mortalidade infantil segundo cor ou raça com base no Censo Demográfico de 2010 e nos sistemas nacionais de informação em saúde no Brasil. Cad. Saúde Pública, Rio de Janeiro, v. 33, n. 7, e00046516, 2017. Disponível em: http://www.scielo.br/scielo. php?script=sci_arttext\&pid=S0102-311X2017000705007\&lng=en\&nrm =iso. Acesso em: 21 out. 2020.

40. GAIVA, M. A. M.; FUJIMORI, E.; SATO, A. P. S. Fatores de risco maternos e infantis associados à mortalidade neonatal. Texto contexto - Enferm., Florianópolis, v. 25, n. 4, e2290015, 2016. Disponível em: http://www.scielo.br/scielo.php?script=sci_arttext\&pid=S0104$-07072016000400318 \&$ Ing=en\&nrm=iso. Acesso em: 21 out. 2020.

41. VICTORA, J. D. et al. Prevalência, mortalidade e fatores de risco associados ao prematuro de muito baixo peso ao nascer: uma análise de 33 anos. J. Pediatr. (Rio J.), Porto Alegre, v. 96, n. 3, p. 327-332, jun. 2020. Disponível em: https://www.scielo.br/scielo.php?pid=S0021$-75572020000300327 \&$ script=sci_arttext\&tlng=pt. Acesso em: 14 nov. 2020.

42. BISMARCK-NASR, E. M.; FRUTUOSO, M. F. P.; GAMABARDELLA, A. M. D. Efeitos tardios do baixo peso ao nascer. Rev Bras. Crescimento Desenvolv. Hum., São Paulo, v. 18, n. 1, p. 98-103, abr. 2008. Disponível em: http://pepsic.bvsalud.org/scielo.php?script=sci_abstract\&pid =S0104-12822008000100013. Acesso em: 30 nov. 2020.

Submetido em: 04/03/2021

Aceito em: 21/06/2021 